Paramagnetism and antiferromagnetic interactions in Cr-doped GaN

This article has been downloaded from IOPscience. Please scroll down to see the full text article.

2011 J. Phys.: Condens. Matter 23346004

(http://iopscience.iop.org/0953-8984/23/34/346004)

View the table of contents for this issue, or go to the journal homepage for more

Download details:

IP Address: 193.136.24.86

The article was downloaded on 12/08/2011 at 15:45

Please note that terms and conditions apply. 


\title{
Paramagnetism and antiferromagnetic interactions in Cr-doped GaN
}

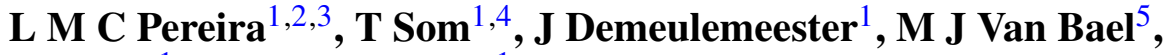 \\ $\mathrm{K}_{\text {Temst }}{ }^{1}$ and A Vantomme ${ }^{1}$ \\ ${ }^{1}$ Instituut voor Kern- en Stralingsfysica and INPAC, K U Leuven, 3001 Leuven, Belgium \\ 2 IFIMUP and IN-Institute of Nanoscience and Nanotechnology, Faculdade de Ciências da Universidade \\ do Porto, 4169-007 Porto, Portugal \\ ${ }^{3}$ Instituto Tecnológico e Nuclear, UFA, 2686-953 Sacavém, Portugal \\ ${ }^{4}$ Institute of Physics, Bhubaneswar 751005, Orissa, India \\ ${ }^{5}$ Laboratory of Solid-State Physics and Magnetism and INPAC, K U Leuven, 3001 Leuven, Belgium
}

E-mail: linomcp@fc.up.pt

Received 8 June 2011, in final form 14 July 2011

Published 12 August 2011

Online at stacks.iop.org/JPhysCM/23/346004

\begin{abstract}
We report on the magnetic and structural properties of $\mathrm{Cr}$-doped $\mathrm{GaN}$ prepared by ion implantation of epitaxial thin films. Based on a detailed analysis of the magnetometry data, we demonstrate that the magnetic interactions between $\mathrm{Cr}$ moments in $\mathrm{GaN}$ are antiferromagnetic (AFM). Increasing the $\mathrm{Cr}$ fractional concentration up to 0.35 , we observe that strong nearest cation neighbor AFM coupling results in the reduction of the effective moment per $\mathrm{Cr}$ atom. The uncompensated $\mathrm{Cr}$ moments exhibit paramagnetic behavior and we discuss to what extent the effects of an anisotropic crystal field and AFM interactions can be inferred from the magnetization data. We discuss the observed changes in magnetic and structural properties induced by thermal annealing in terms of defect annealing and $\mathrm{Cr}$ aggregation.
\end{abstract}

\section{Introduction}

Over the past decade, the magnetism of wide-gap semiconductors doped with $3 d$ transition metals, or dilute magnetic semiconductors (DMS), has become one of the most controversial topics in condensed matter physics. Despite the remarkable volume of experimental and theoretical research and significant developments in synthesis and characterization methods, the origin of high-temperature ferromagnetism in wide-gap DMS remains far from consensual [1].

Soon after the first report on Co-doped $\mathrm{TiO}_{2}$ [2], ferromagnetism at and above room temperature was reported in many other wide-gap DMS materials (cf, e.g. the reviews [3-6]). Research in the ensuing years followed two main directions, the search (i) for new DMS materials displaying signatures of high-temperature ferromagnetic order (e.g. Gd-doped GaN [7], C-doped $\mathrm{ZnO}$ [8], and undoped $\mathrm{HfO}_{2}$ [9]) and (ii) for the origin and control of ferromagnetism in those systems regarded as most attractive (e.g. Co- and Mn-doped $\mathrm{ZnO}$ [10] and Mn-doped GaN [11]). However, as the understanding of some of the most intensively studied materials deepened, issues of irreproducibility and instability became common features of these materials. A number of non-intrinsic sources of ferromagnetism were identified and became well documented: magnetic contaminations [12-15], measurement artifacts [13, 14], spinodal decomposition (e.g. in Co-doped $\mathrm{ZnO}$ [16]) and secondary-phase formation (e.g. in $\mathrm{ZnO}$ doped with $\mathrm{Fe}$, $\mathrm{Co}$ and $\mathrm{Ni}$ [17-19]). On the other hand, studies on well-characterized DMS materials (single-phase and homogeneous) found only paramagnetism (e.g. in Mn-doped GaN [20] and Co-doped $\mathrm{ZnO}$ [21]), antiferromagnetic interactions (e.g. in Mn-doped GaN [22] and in Co-doped $\mathrm{ZnO}$ [23-26]), or, at best, ferromagnetic order with very low Curie temperature (e.g. in Mn-doped GaN [11]). Consequently, the current view on wide-gap DMS is moving toward the belief that the often observed high-temperature ferromagnetism may be non-intrinsic [1]. Key to solving this controversy is a systematic and detailed mapping of the type and strength of the magnetic interactions in the most representative combinations of magnetic dopants and wide-gap semiconductor hosts. In this sense, $\mathrm{Cr}$-doped $\mathrm{GaN}$ is an interesting case. Although far from being one of the most intensively studied cases, it has accumulated a significant number of reports of 
high-temperature ferromagnetism [27-33]. However, while focusing on the intrinsic origin of the observed ferromagnetic signatures by demonstrating the absence of secondary-phase formation, these studies lack a deeper analysis of the magnetic behavior itself.

Transition metals can be incorporated into GaN during growth [27-31] or by ion implantation [32, 33]. The advantage of ion implantation with respect to the production of uniform and single-crystalline DMS nanolayers is that it is less likely to suffer from the formation of secondary phases. Ion bombardment inevitably leads to lattice disorder, a major concern when electrical and optical dopants are introduced by ion implantation [34]. For magnetic doping, however, lattice disorder is believed to be crucial in establishing the ferromagnetic order, in the form of either point [35] or extended [36] defects. Depending on the implantation conditions, different types of defects can be produced in ion-implanted GaN. At the low fluence end, isolated [37] or clustered [38] point defects are typically observed. As the fluence increases, bands of large planar defects are formed parallel to the basal plane of GaN [38]. Ion-implanted DMS materials are thus interesting systems in which to study the interplay between magnetic dopants and lattice defects.

The most straightforward and reproducible parameter to control, in order to tune the amount and type of ion beam damage, is temperature, either during implantation or during subsequent thermal annealing [39]. Typically, the accumulation of stable defects in irradiated semiconductors, which can eventually result in amorphization, can be reduced by increasing the implantation temperature. In $\mathrm{GaN}$, the accumulated damage decreases rather sharply as the irradiation temperature is increased up to $300^{\circ} \mathrm{C}$, and more slowly above that [40]. On the other hand, high fluence ion implantation at elevated temperatures $\left(550^{\circ} \mathrm{C}\right)$ has been reported to induce dramatic erosion of $\mathrm{GaN}$ [41]. Implantation temperatures around $300{ }^{\circ} \mathrm{C}$ can thus be expected to yield a good compromise between avoiding amorphization and minimizing surface erosion of GaN. Further control over beam-induced damage is typically realized by thermal treatment. Annealing of implantation disorder in $\mathrm{GaN}$ is, however, a rather complex problem. The rule of thumb is that temperatures of around two thirds of the melting point (in units of $\mathrm{K}$ ) are required to remove extended defects in compound semiconductors. For $\mathrm{GaN}$, this corresponds to about $1600{ }^{\circ} \mathrm{C}$, based on its calculated melting point [42]. However, annealing of $\mathrm{GaN}$ at such high temperatures is counteracted by material decomposition involving loss of nitrogen from the $\mathrm{GaN}$ surface (e.g. [43]). In addition, hightemperature annealing may result in undesired segregation of $\mathrm{Cr}$ or $\mathrm{CrN}$ secondary phases [44]. Therefore, when studying the magnetism of ion-implanted GaN DMS materials and its dependence on defect concentration, optimum annealing conditions are those providing a significant degree of damage recovery while avoiding surface decomposition and phase segregation.

In this paper, we analyze the magnetic and structural properties of $\mathrm{Cr}$-doped $\mathrm{GaN}$ thin films, produced by implanting $\mathrm{Cr}^{+}$ions in epitaxial $\mathrm{GaN}$ thin films. Covering
Table 1. Sample list. The naming is according to substrate, $\mathrm{S}$ for silicon and $\mathrm{A}$ for $\mathrm{Al}_{2} \mathrm{O}_{3}$, and implanted fluence, in units of $1 \times 10^{15}$ at. $\mathrm{cm}^{-2}$. All implantations were performed at $300^{\circ} \mathrm{C}$.

\begin{tabular}{llll}
\hline Name & Substrate & Fluence $($ at. cm \\
\hline S1 & $\mathrm{Si}$ & $1 \times 10^{15}$ & Peak concentration $\left(x_{\mathrm{p}}\right)$ \\
$\mathrm{A} 1$ & $\mathrm{Al}_{2} \mathrm{O}_{3}$ & $1 \times 10^{15}$ & 0.005 \\
$\mathrm{~S} 4$ & $\mathrm{Si}$ & $4 \times 10^{15}$ & 0.005 \\
$\mathrm{~A} 4$ & $\mathrm{Al}_{2} \mathrm{O}_{3}$ & $4 \times 10^{15}$ & 0.02 \\
$\mathrm{~S} 10$ & $\mathrm{Si}$ & $1 \times 10^{16}$ & 0.02 \\
$\mathrm{~A} 10$ & $\mathrm{Al}_{2} \mathrm{O}_{3}$ & $1 \times 10^{16}$ & 0.05 \\
$\mathrm{~S} 20$ & $\mathrm{Si}$ & $2 \times 10^{16}$ & 0.05 \\
$\mathrm{~A} 20$ & $\mathrm{Al}_{2} \mathrm{O}_{3}$ & $2 \times 10^{16}$ & 0.10 \\
$\mathrm{~S} 40$ & $\mathrm{Si}$ & $4 \times 10^{16}$ & 0.10 \\
$\mathrm{~A} 40$ & $\mathrm{Al}_{2} \mathrm{O}_{3}$ & $4 \times 10^{16}$ & 0.20 \\
$\mathrm{~S} 70$ & $\mathrm{Si}_{\mathrm{Ai}}$ & $7 \times 10^{16}$ & 0.20 \\
$\mathrm{~A} 70$ & $\mathrm{Al}_{2} \mathrm{O}_{3}$ & $7 \times 10^{16}$ & 0.35 \\
\hline
\end{tabular}

a wide range of $\mathrm{Cr}$ fractional concentrations (0.005-0.35), from the very dilute regime to well above the site percolation threshold (0.20), and focusing on an in-depth analysis of the magnetometry data, we aim at identifying the type of magnetic interactions between $\mathrm{Cr}$ moments in $\mathrm{GaN}$. Combined with structural characterization of as-implanted and annealed samples by means of x-ray diffraction (XRD) and Rutherford backscattering and channeling spectrometry $(\mathrm{RBS} / \mathrm{C})$, we investigate how the magnetic behavior is influenced by thermal annealing.

\section{Experimental results}

\subsection{Sample preparation and structural characterization}

High-quality epitaxial $\mathrm{GaN}(0001)$ thin films were grown by metalorganic vapor phase epitaxy (MOVPE). Two wafers were grown using different substrates: a $2 \mu \mathrm{m}$ thick $\mathrm{GaN}(0001)$ layer on an $\mathrm{Al}_{2} \mathrm{O}_{3}(0001)$ substrate and an $800 \mathrm{~nm}$ $\mathrm{GaN}(0001)$ layer on a $\mathrm{Si}(111)$ substrate using an AlN/AlGaN intermediate layer. Pieces from each of the wafers were implanted at $300{ }^{\circ} \mathrm{C}$ with $\mathrm{Cr}^{+}$ions with an energy of $80 \mathrm{keV}$ to fluences ranging from $1 \times 10^{15}$ to $7 \times 10^{16}$ at. $\mathrm{cm}^{-2}$. The implantations were performed under an angle of $7^{\circ}$ with respect to the surface normal in order to minimize ion channeling [45]. Using the TRIM code [46] to simulate the Gaussian-like ion distribution, we estimated a projected range of $R_{\mathrm{P}}=38 \mathrm{~nm}$, a longitudinal straggling of $17 \mathrm{~nm}$ and peak concentrations $\left(x_{\mathrm{p}}\right)$ ranging from 0.005 to 0.35 . Table 1 lists the implanted samples and the corresponding fluences and $x_{\mathrm{p}}$. All samples were studied in the as-implanted state and after thermal annealing at $800^{\circ} \mathrm{C}$ in $\mathrm{N}_{2}$ flow for $2.5 \mathrm{~h}$, using a GaN proximity cap to minimize surface degradation. In order to avoid sample contamination with ferromagnetic impurities [15], the implantations were carried out using a Mo sample holder and the samples were placed in a ceramic boat during annealing. Each entry in table 1 corresponds to two samples, one for the structural characterization and the other for magnetometry measurements. The samples reserved for the magnetic characterization were handled with non-metallic tweezers in order to avoid magnetic contamination [15]. 

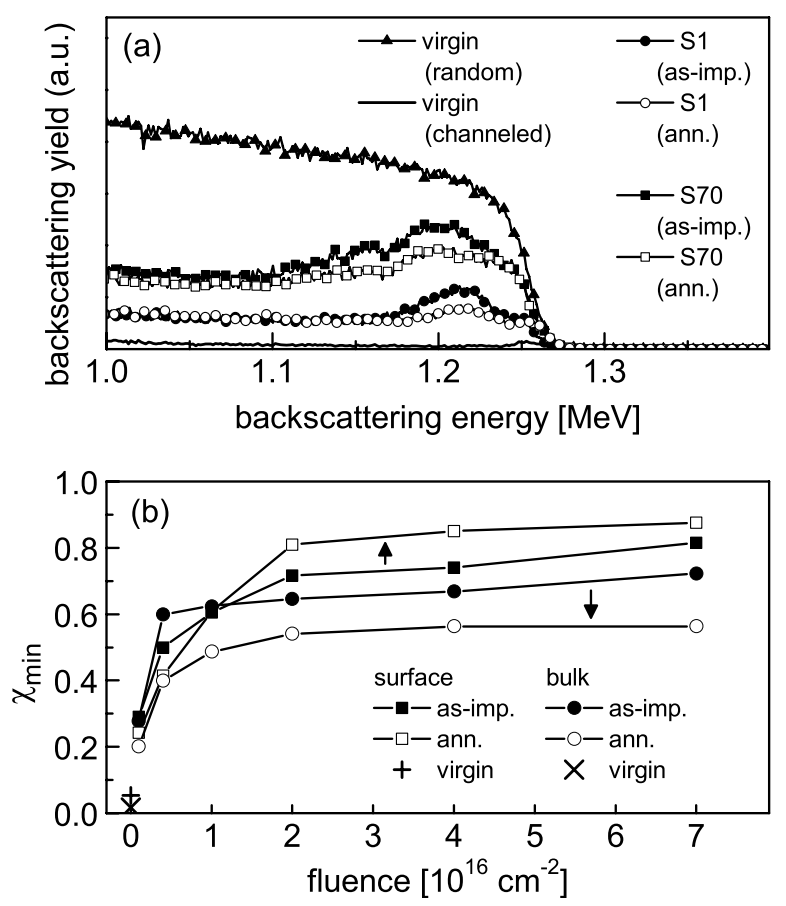

Figure 1. (a) Selected RBS/C spectra: random and channeled spectra of a virgin (unimplanted) GaN sample and channeled spectra of the samples with the lowest $\left(\mathrm{S} 1,1 \times 10^{15} \mathrm{~cm}^{-2}\right)$ and the highest (S70, $\left.7 \times 10^{16} \mathrm{~cm}^{-2}\right)$ implanted fluences, before and after annealing at $800^{\circ} \mathrm{C}$. (b) Minimum yield $\left(\chi_{\min }\right)$ determined from both the near-surface and bulk peaks of disorder, before and after annealing, as a function of implanted fluence.

Defect formation and recovery were studied using $\mathrm{RBS} / \mathrm{C}$, with a $1.57 \mathrm{MeV} \mathrm{He}^{+}$beam. Figure 1 shows examples of spectra obtained in random and channeled geometries (a) and summarizes the results in terms of damage accumulation and recovery in both the near-surface (first few monolayers) and bulk (end of range) regions (b). It is clear that implanting at $300^{\circ} \mathrm{C}$ successfully suppresses amorphization, as both near-surface and bulk disorder exhibit saturation at levels which are considerably below the random level. In addition, there is no sign of dramatic surface erosion. This can be inferred from the position of the bulk damage peak relative to the surface. If the film erosion had reached depths of the order of the ion range, the bulk damage peak would have shifted toward the surface as has been reported in [47]. Furthermore, within the experimental uncertainty $(<30 \mathrm{~nm})$ the film thickness does not decrease after implantation, even for the maximum fluence. Showing that film erosion was minimal has two important practical consequences. Not only does it show that the nominal $\mathrm{Cr}$ content was conserved, it also validates the simulated $\mathrm{Cr}$ depth profiles and peak concentrations, calculated assuming a constant film thickness. Regarding the choice of annealing treatment, figure 1 also shows that while providing a significant reduction of the lattice disorder in the Cr-doped region (depicted by the $\downarrow$ in figure 1(b)) it kept the near-surface damage peak below random levels.

The possible formation of $\mathrm{Cr}$ or $\mathrm{CrN}$ precipitates was investigated using conventional XRD. Other than those associated with the GaN layers and the substrate, no additional peaks could be resolved, either before or after annealing (data not shown). Only typical signs of reduced crystalline quality due to implantation were observed. Although thermal annealing of similar wide-gap semiconductors implanted with transition metals can result in the formation of small precipitates which are difficult to detect with conventional XRD (e.g. Co-, Ni- and Fe-implanted $\mathrm{ZnO}$ in $[18,19,17]$ ), $\mathrm{Cr}$ was found to be very stable in substitutional sites in $\mathrm{GaN}$, at least up to annealing temperatures of $825^{\circ} \mathrm{C}$ [31]. The presence of $\mathrm{Cr}$ or $\mathrm{CrN}$ precipitates in our samples is thus highly unlikely, both before and after annealing $\left(800^{\circ} \mathrm{C}\right)$. However, although very unlikely in the as-implanted state, the formation of (substitutional) Cr-rich regions during annealing cannot be excluded. Such substitutional clusters would be coherent with the GaN matrix and thus difficult to detect by diffraction techniques. This chemical phase separation by aggregation of the transition-metal dopants, known as spinodal decomposition in the DMS literature, has been observed in a number of DMS materials, including Cr- [44] and Fe-doped GaN [48].

From the structural characterization described in this section we can conclude that, in terms of implantation conditions, we have successfully avoided both amorphization and significant surface erosion. Regarding the annealing treatment, while inducing a significant degree of lattice recovery, we have avoided the formation of secondary phases, at least within the sensitivity of conventional XRD. However, the formation of nanoprecipitates and substitutional Cr clusters cannot be excluded.

\subsection{Magnetic properties}

The magnetic characterization was performed using a superconducting quantum interference device (SQUID) magnetometer (Quantum Design MPMS XL-5) following strict procedures in order to avoid measurement artifacts and external magnetic contributions. These procedures were developed based on statistically relevant tests, which allowed us to determine the practical limits of SQUID magnetometry for the detection of ferromagnetism under various sample preparation, processing and handling conditions [15]. Unless otherwise specified, the measurements shown and discussed below were performed with the field perpendicular to the $\mathrm{GaN}$ $c$-axis, i.e. parallel to the film plane.

2.2.1. Absence of ferromagnetic order. A small hysteresis, with a saturation moment always below $1 \times 10^{-6} \mathrm{emu}$, was observed in some of the samples, up to room temperature. No correlation was found with respect to the fluence or annealing conditions. Since the same residual ferromagneticlike behavior was observed in unimplanted control samples, we attribute it to small ferromagnetic contaminations [15]. As such, we can conclude the following.

Finding I. None of the samples, regardless of substrate, fluence or annealing treatment, showed ferromagnetic behavior above the range dominated by ferromagnetic contaminations (i.e. with saturation moments $>1 \times 10^{-6} \mathrm{emu}$ ), from $300 \mathrm{~K}$ down to $2 \mathrm{~K}$. 


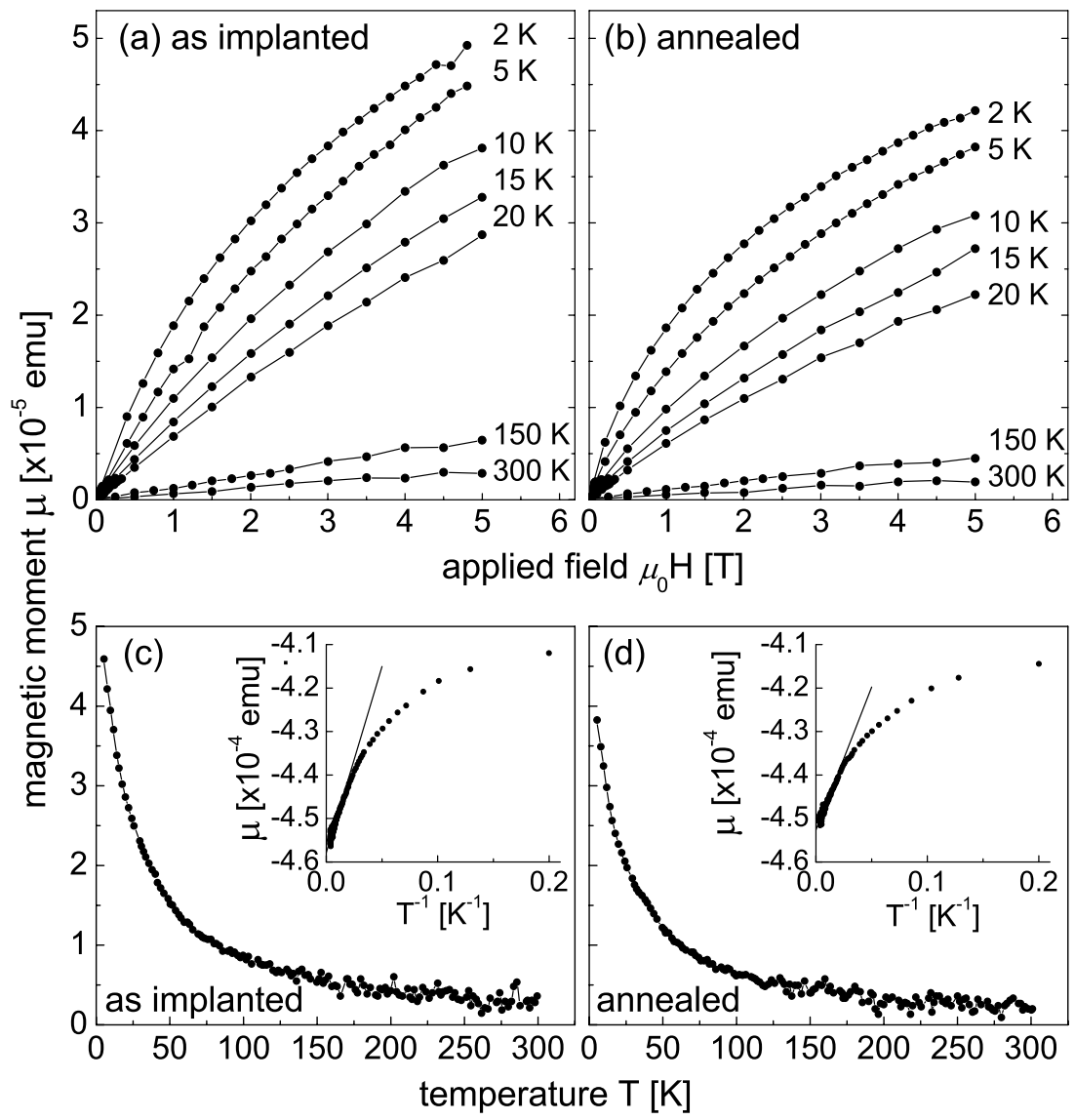

Figure 2. Representative set of SQUID measurements, for sample A70 $\left(x_{\mathrm{p}}=0.35\right)$ before ((a) and (c)) and after ((b) and (d)) annealing: $M-H$ data ((a) and (b)) at 2, 5, 10,15, 20, 150 and $300 \mathrm{~K}$ and $M-T$ data ((c) and (d)) for an applied field of $5 \mathrm{~T}$, all corrected for the diamagnetic background. Insets: uncorrected $M-T$ data of (c) and (d) plotted versus $T^{-1}$ with a linear fit between 100 and $300 \mathrm{~K}$.

Since intrinsic irreversibility was not observed in any of the samples, only one quarter of each of the $M-H$ loops (magnetization as a function of applied field) is shown in the remainder of this paper, i.e. only the data corresponding to positive and decreasing field.

As a representative example, a typical set of measurements is shown in figure 2 for sample A70 $\left(x_{\mathrm{p}}=0.35\right)$ before ((a) and (c)) and after ((b) and (d)) annealing. The magnetic moment $(\mu)$ was measured as a function of applied field $(M-H)$ up to $5 \mathrm{~T}$ at temperatures between 2 and $300 \mathrm{~K}$ ((a) and (b)) and as a function of temperature $(M-T)$ between 5 and $300 \mathrm{~K}$ with an applied field of $5 \mathrm{~T}((\mathrm{c})$ and (d)). All the data in figure 2 are corrected for the diamagnetic component (dominated by the substrate) and exhibit typical paramagnetic behavior. This diamagnetic background was estimated from a linear fit to $\mu\left(T^{-1}\right)$ above $100 \mathrm{~K}$ (insets of (c) and (d)), assuming a Curie behavior of the paramagnetic component and a temperature independent diamagnetic term

$$
\chi=\frac{\mu}{H}=\chi_{\mathrm{C}}+\chi_{\mathrm{DM}}=\frac{C}{T}+\chi_{\mathrm{DM}},
$$

where $\chi$ is the total magnetic susceptibility, $\chi_{\mathrm{C}}$ the Curie term, $\chi_{\mathrm{DM}}$ the diamagnetic term, $C$ the Curie constant and $T$ the temperature. The diamagnetic susceptibility was further corrected by subtracting the very small contribution of ferromagnetic contaminations (when present), estimated from the $M-H$ data at $300 \mathrm{~K}$ between 2 and $5 \mathrm{~T}$ (in saturation). The result was checked for consistency by comparing it with the diamagnetic susceptibility obtained from the linear fit to the $M-H$ data at 150 and $300 \mathrm{~K}$, taking into account the paramagnetic component (linear at $T>100 \mathrm{~K}$ to a good approximation). The $\mathrm{Si}$ substrates, both before and after deposition of the GaN thin films, showed a small temperature dependence of $\chi_{\mathrm{DM}}$, most likely due to Van Vleck paramagnetism, which has also been observed in other semiconductor substrates, e.g. GaAs [49]. The $\chi_{\mathrm{DM}}$ value used to correct the $M-H$ data of the samples grown on $\mathrm{Si}$ was itself corrected for this small temperature dependence, based on measurements of unimplanted samples (as both the diamagnetic and the Van Vleck paramagnetic susceptibilities are extensive properties, i.e. scale with the substrate's mass). We note that, in general, the paramagnetic contribution does not necessarily follow the Curie term in equation (1), but rather a Curie-Weiss law

$$
\chi_{\mathrm{CW}}=\frac{C}{T-\Theta},
$$

where $\Theta$ is the Weiss constant which accounts for magnetic interactions. Nonetheless, equation (1) holds for $|\Theta / T| \ll 1$, which we show below to be the case in our data. From this point on, all the data that are shown or referred to were corrected for the diamagnetic substrate background 

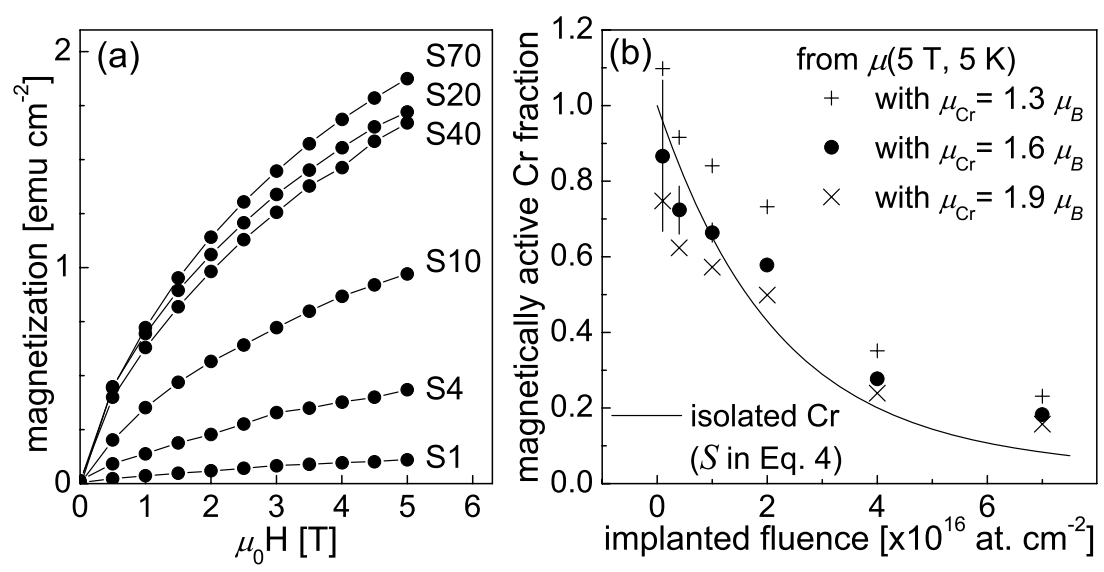

Figure 3. (a) $M-H$ at $5 \mathrm{~K}$, in units of emu per unit of the film surface area, after annealing, for the films grown on Si. Note that full saturation is not reached. (b) Fraction of magnetically active $\mathrm{Cr}$ atoms, assuming different values of moment per atom $\left(\mu_{\mathrm{Cr}}\right)$ at $5 \mathrm{~K}$ and $5 \mathrm{~T}$ and fraction of isolated $\mathrm{Cr}$ atoms estimated using equation (4) (line), as a function of implanted fluence. The best fit is obtained for $\mu_{\mathrm{Cr}}=1.6 \mu_{\mathrm{B}}(\bullet)$; the data are also shown for $\mu_{\mathrm{Cr}}=1.3 \mu_{\mathrm{B}}(+)$ and $\mu_{\mathrm{Cr}}=1.9 \mu_{\mathrm{B}}(\times)$ for comparison. Note that $\mu_{\mathrm{Cr}}=1.6 \mu_{\mathrm{B}}$ is the non-saturated moment per $\mathrm{Cr}$ atom (at $5 \mathrm{~K}$ and $5 \mathrm{~T}$ ).

using this method. This was found to be more accurate than, for example, subtracting $M-H$ data measured prior to implantation. Small differences in sample mounting can result in a change of a few per cent in the total measured moment. Since the diamagnetic signal largely dominates, the curves resulting from this subtraction would be affected by an erroneous linear component. At least qualitatively, all the samples show a behavior similar to that of sample S70 (figure 2). We can thus conclude the following.

Finding II. All samples exhibit paramagnetic behavior.

Before discussing the details of this paramagnetism in terms of field and temperature dependence, we will describe how the effective moment per $\mathrm{Cr}$ atom evolves with increasing Cr concentration.

2.2.2. Cr moment quenching with increasing Cr concentration. Figure 3(a) shows the magnetization at $5 \mathrm{~K}$, for the implanted and annealed $\mathrm{GaN}$ samples grown on $\mathrm{Si}$ (the results are equivalent for the $\mathrm{Al}_{2} \mathrm{O}_{3}$ series). The magnetization does not increase linearly with $\mathrm{Cr}$ concentration; there is a clear saturation for fluences above $2 \times 10^{16} \mathrm{~cm}^{-2}$, which, based on the RBS/C data, cannot be ascribed to a saturation of the $\mathrm{Cr}$ content due to film erosion. We attribute this effect to the antiparallel alignment of the magnetic moments of $\mathrm{Cr}$ atoms sitting in nearest cation neighbor sites, due to strong antiferromagnetic (AFM) interactions. A similar moment quenching effect has been observed in other DMS systems, e.g. Co-doped $\mathrm{ZnO}[23,24]$. To model this behavior, we assume that all $\mathrm{Cr}$ impurities randomly occupy Ga sites [31, $50,51]$ and that only isolated $\mathrm{Cr}$ atoms, i.e. without $\mathrm{Cr}$ nearest cation neighbors in the Ga sublattice, effectively contribute to the magnetization. The fraction of isolated $\mathrm{Cr}$ atoms in the GaN hexagonal lattice $\left(S^{\prime}\right)$ can be estimated using Behringer's equation

$$
S^{\prime}=(1-x)^{12}
$$

where $x$ is the fractional concentration of $\mathrm{Cr}$ atoms [52]. Since in implanted samples $x$ is a function of depth $l$, one must integrate $S^{\prime}$ over the film thickness as

$$
S=\int_{0}^{\infty} p(l) S^{\prime}(l) \mathrm{d} l,
$$

with

$$
p(l)=\frac{x(l)}{\int_{0}^{\infty} x(l) \mathrm{d} l},
$$

where $x(l)$ is the concentration profile which we have simulated using TRIM. Assuming that each of the magnetically active $\mathrm{Cr}$ atoms contributes with a moment $\mu_{\mathrm{Cr}}$ for a given field and temperature, one can extract the corresponding fraction from the experimental data and compare it to that of isolated $\mathrm{Cr}$ estimated using equation (4). Figure 3(b) shows the fraction of magnetically active $\mathrm{Cr}$ atoms, using $\mu_{\mathrm{Cr}}=1.6 \mu_{\mathrm{B}}$ (at $5 \mathrm{~K}$ and $5 \mathrm{~T}$ ), which yields the best fit to equation (4). The agreement is fairly good, particularly considering that $\mu_{\mathrm{Cr}}$ is the only free parameter. Note that $1.6 \mu_{\mathrm{B}}$ is the non-saturated moment per $\mathrm{Cr}$ atom, which can explain why it is smaller than the expected $3 \mu_{\mathrm{B}}$ for the high-spin $\mathrm{Cr}^{3+}\left(3 d^{3}\right)$ state, predicted by Hund's rules and by $a b$ initio calculations [53]. At high concentration, the model somewhat underestimates the active $\mathrm{Cr}$ fraction. This is likely to result from considering the simplest case for which only the isolated $\mathrm{Cr}$ atoms contribute to the magnetization. For low concentrations, the majority of the non-isolated $\mathrm{Cr}$ atoms are in the form of pairs (dimers). Such a dimer configuration may indeed produce an almost perfect antiparallel alignment of the two magnetic moments. However, for higher concentrations, larger complexes of three and more atoms are formed. For these larger substitutional clusters, particularly those containing an odd number of Co atoms, the quenching of the $\mathrm{Cr}$ moment may be less efficient due to frustration effects and the presence of uncompensated moments, which may thus contribute to the measured magnetization. This discussion can be concluded as follows. 


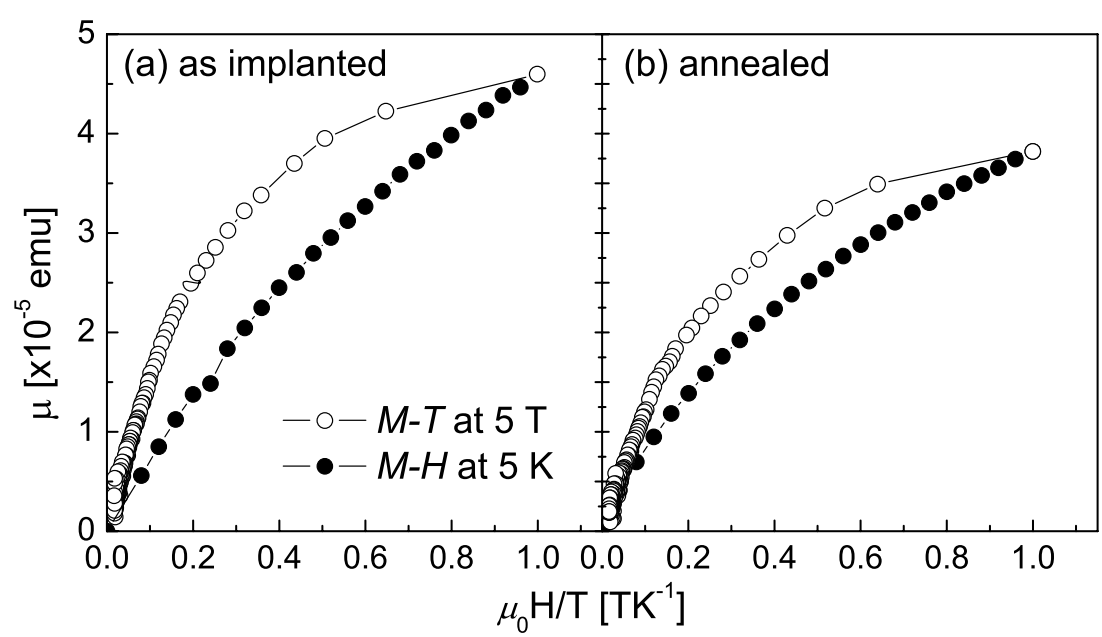

Figure 4. Magnetic moment plotted versus $H / T$ for sample A70 ( $\left.x_{\mathrm{p}}=0.35\right)$ before (a) and after (b) annealing, using $M-T$ data between 5 and $300 \mathrm{~K}$ with an applied field of $5 \mathrm{~T}(\mathrm{\circ})$ and $M-H$ data at $5 \mathrm{~K}$ up to $5 \mathrm{~T}(\bullet)$. As expected, the data do not overlap, i.e. the magnetization does not follow a Brillouin behavior.

Finding III. Strong AFM interactions between the Cr atoms in nearest cation neighbor complexes impose the antiparallel alignment of the corresponding magnetic moments. This results in a $\mathrm{Cr}$ moment quenching effect, i.e. a decrease of the effective moment per $\mathrm{Cr}$ atom with increasing $\mathrm{Cr}$ concentration.

2.2.3. Paramagnetism. While I, II and III constitute the major findings of this work, it is worth discussing in more detail the paramagnetism of the uncompensated (or not fully compensated) $\mathrm{Cr}$ moments in $\mathrm{GaN}$ and comparing it to the intensively studied and thus better understood wide-gap DMS materials Mn-doped GaN and Co-doped $\mathrm{ZnO}$.

The magnetization of ideal non-interacting magnetic moments with constant total angular momentum quantum number $J$ in a magnetic field $H$ follows the Brillouin function $B_{J}(H, T)$. This is obviously not the case for $\mathrm{Cr}$ moments in $\mathrm{GaN}$, which are subject to (1) the anisotropic GaN crystal field of the host and, as we have shown above, (2) magnetic interactions. The non-Brillouin behavior of our samples is easily confirmed by plotting the $M-H$ and $M-T$ data together as a function of $H / T$ (figure 4). As expected, contrary to what would happen for an ideal Brillouin paramagnet $\left(\mu(H, T, J) \propto B_{J}(H / T)\right)$, the curves do not overlap. In the following, we discuss to what extent we find evidence of both effects, (1) anisotropic crystal field and (2) magnetic interactions, in Cr-implanted GaN.

The most easily observable effect of an anisotropic crystal field (effect (1)) on a paramagnetic $3 d$ moment is the resulting anisotropic magnetization. The trigonal crystal field in the wurtzite structure induces a magnetic anisotropy via the spin-orbit interaction, which can be described by an effective spin Hamiltonian with a zero field splitting constant $D$ and an anisotropic effective $g$-factor $\left(g_{\|}\right.$for parallel and $g_{\perp}$ for perpendicular magnetic field with respect to the $c$-axis). A strongly anisotropic magnetization was observed, for example, for $\mathrm{Mn}^{3+}$ ions in $\mathrm{GaN}$ [20] and $\mathrm{Co}^{2+}$ ions in $\mathrm{ZnO}$ [21], and successfully described by well-established values of the phenomenological parameters $D, g_{\|}$and $g_{\perp}[21]$ or more detailed Hamiltonians [20]. Figure 5 shows $M-H$ and $M-T$ data of sample A70 $\left(x_{\mathrm{p}}=0.35\right)$ for field applied parallel and perpendicular to the sample plane, i.e. perpendicular and parallel to the $\mathrm{GaN} c$-axis, respectively. Although some degree of anisotropy can be resolved, the effect is much weaker than those observed in Mn-doped GaN [20] and Co-doped $\mathrm{ZnO}$ [21]. Because the parameters $D, g_{\|}$and $g_{\perp}$ have never been determined for $\mathrm{Cr}$ impurities in $\mathrm{GaN}$ or similar materials, one cannot predict the magnetic anisotropy for Cr-doped $\mathrm{GaN}$ under these experimental conditions. Nonetheless, the fact that the effect is so small in our samples can be explained, at least partially, by the significant disorder induced by implantation (section 2.1), which disturbs the crystal periodicity and consequently the crystal field anisotropy.

In general, the presence of magnetic interactions (effect (2)) can be inferred from magnetization data as a perturbation to the paramagnetic behavior above the associated ordering temperature. In principle, this can be modeled using an effective temperature $(T-\Theta)$ where $\Theta$ accounts for the magnetic interactions [54], which has been done, for example, for Mn-doped GaN [22] and Co-doped $\mathrm{ZnO}$ [24-26]. Applying a similar approach to our data, figure 6 shows the $\mu^{-1}(T)$ data of sample A70 $\left(x_{\mathrm{p}}=0.35\right)$ before and after annealing and a fit to the Curie-Weiss law equation (2), which corresponds to the Curie law with an effective temperature $T-\Theta$. The data were fitted only between 60 and $100 \mathrm{~K}$ to avoid a possible contribution from frozen $\mathrm{O}_{2}$ between 30 and $60 \mathrm{~K}$ and the large data dispersion above $100 \mathrm{~K}$. The fit yields a negative $\Theta,-15(3) \mathrm{K}$ and $-5(3) \mathrm{K}$ before and after annealing, respectively, which can in principle be interpreted as a signature of AFM interactions. We note, however, that this type of analysis should be applied very carefully. In [21] it is shown that, while the paramagnetism of Co-doped $\mathrm{ZnO}$ is correctly modeled using an adequate effective spin Hamiltonian without considering magnetic interactions, attempting to fit the same data using inadequate models such as the Brillouin function or the 


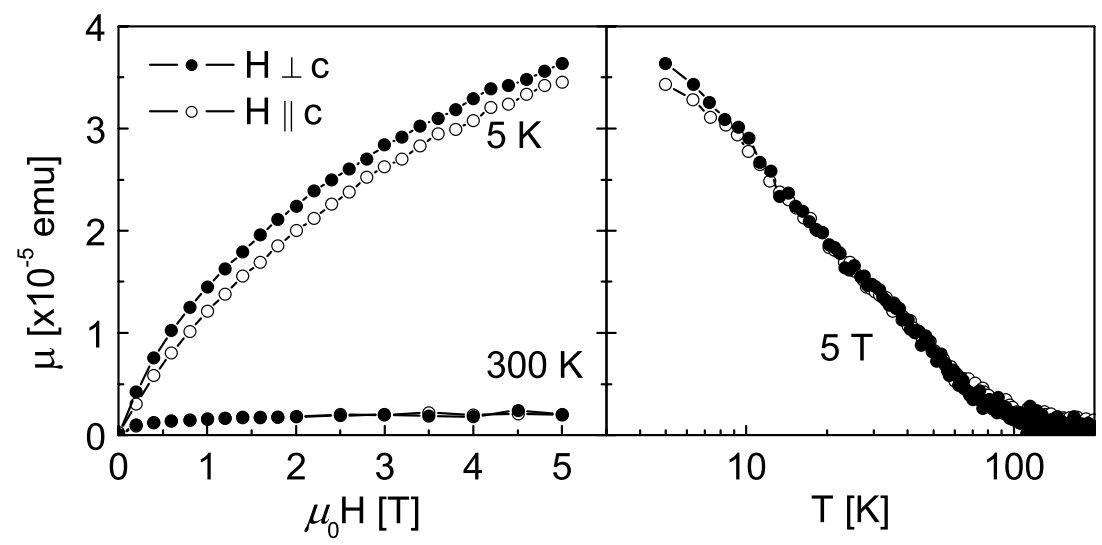

Figure 5. Comparison of the measured magnetization of sample $\mathrm{S} 70\left(x_{\mathrm{p}}=0.35\right)$, after annealing, for magnetic field applied perpendicular (•) and parallel (o) to the $\mathrm{GaN} c$-axis: (a) $M-H$ data at $5 \mathrm{~K}$; (b) $M-T$ data with an applied field of $5 \mathrm{~T}$.

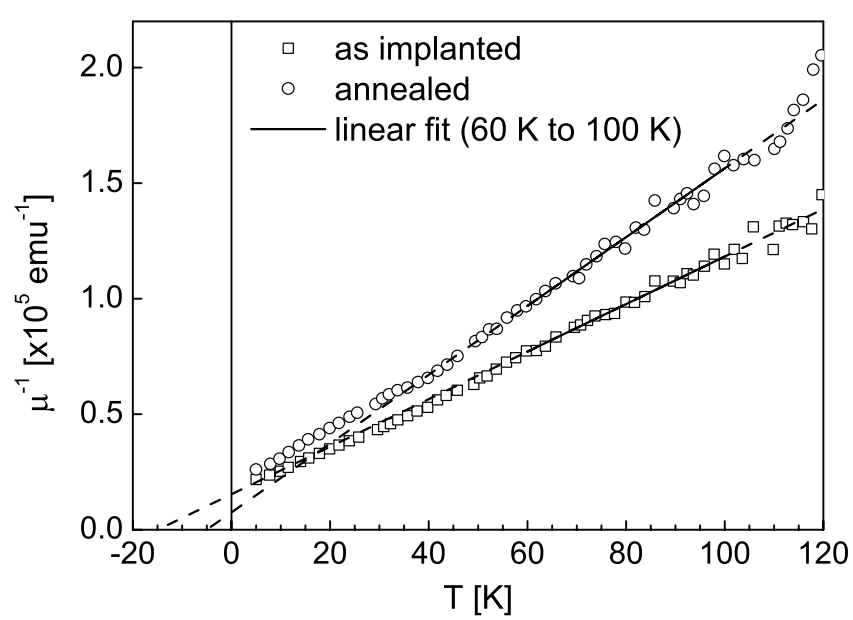

Figure 6. $\mu^{-1}(T)$ data of sample $\mathrm{A} 70\left(x_{\mathrm{p}}=0.35\right)$ before and after annealing and a Curie-Weiss fit after equation (2) between 60 and $100 \mathrm{~K}$ (line). The results of the fit are discussed in the text in terms of AFM interactions and crystal field effects.

Curie law would require the use of an effective temperature $T-\Theta\left(T+T_{0}\right.$ in [21]), which could be misinterpreted as an effect of antiferromagnetic interactions. Nevertheless, in our case, because some of the $\mathrm{Cr}$ atoms in nearest cation neighbor complexes are not fully compensated, i.e. have a finite contribution to the magnetization (section 2.2.2), it is reasonable to conclude that $\Theta$ contains at least a contribution from the nearest cation neighbor AFM interactions.

Finally we will briefly discuss the effect of thermal annealing on the magnetic behavior. We have shown in figure 6 that $|\Theta|$ decreases from $-15(3)$ to $-5(3) \mathrm{K}$ upon thermal annealing, indicating a change in crystal field (1) or a weakening of the AFM interactions (2) or a combination of both. The changes in magnetic behavior induced by thermal annealing are even more evident in the $M-H$ data. This is illustrated in figure 7 where we show the 5, 20 and $300 \mathrm{~K}$ $M-H$ data for samples S20 $\left(x_{\mathrm{p}}=0.10\right)$ and A70 $\left(x_{\mathrm{p}}=\right.$ 0.35 ): consistently throughout the entire concentration range, thermal annealing reduces the magnetization, except for very low temperatures and fields where it does the opposite. Structurally, the annealing can have two effects which may explain these changes in magnetic behavior.

(i) Annealing of lattice defects as demonstrated by RBS/C above. Lattice defects in the vicinity of $\mathrm{Cr}$ atoms modify the crystal field (1) and may even mediate magnetic
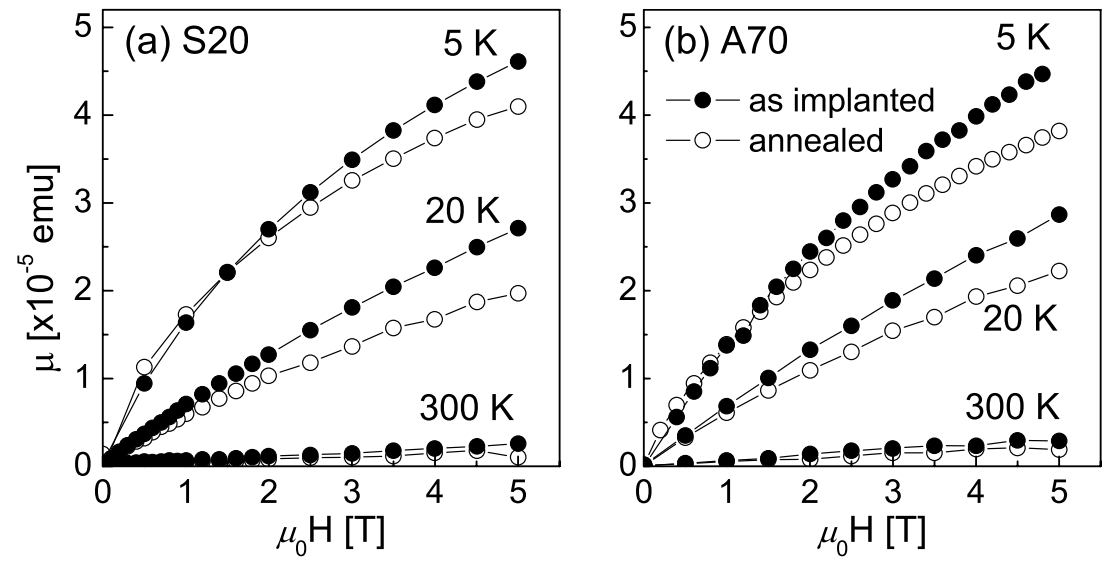

Figure 7. $M-H$ data at 5,20 and $300 \mathrm{~K}$ for samples $\mathrm{S} 20\left(x_{\mathrm{p}}=0.10\right)(\mathrm{a})$ and $\mathrm{A} 70\left(x_{\mathrm{p}}=0.35\right)(\mathrm{b})$, before $(\bullet)$ and after $(\circ)$ annealing. 
interactions between distant $\mathrm{Cr}$ moments (2). Some point defects may even contribute to the magnetization directly. For example, it has been predicted that $\mathrm{Ga}$ vacancies induce non-vanishing magnetic moments in the neighboring $\mathrm{N}$ atoms [55] and that $\mathrm{N}$ vacancies enhance the magnetic moment of isolated $\mathrm{Cr}$ impurities [53, 56].

(ii) Aggregation of $\mathrm{Cr}$ atoms in substitutional clusters or in small precipitates of $\mathrm{Cr}$ or $\mathrm{CrN}$ which may be undetectable by XRD. By modifying the dopant spatial distribution, i.e. the relative distance between $\mathrm{Cr}$ atoms and the local arrangement of their neighbors, $\mathrm{Cr}$ aggregation would also affect the crystal fields (1) and the magnetic interactions (2).

In short, thermal annealing can induce two distinct structural modifications ((i) and (ii)), each of which can modify each of the two parameters which control the magnetic behavior ((1) and (2)). Such an intricate interplay complicates a more quantitative approach to this discussion, which we thus simply conclude as follows.

Finding IV. Uncompensated $\mathrm{Cr}$ atoms behave as paramagnetic moments perturbed by AFM interactions in an anisotropic crystal field. Although minimally, thermal annealing affects this paramagnetism, possibly due to an interplay between defect annealing and $\mathrm{Cr}$ aggregation.

\section{Discussion}

The main finding of this study is that the magnetic interactions between $\mathrm{Cr}$ moments in $\mathrm{GaN}$ are not ferromagnetic but AFM, which is in contrast with previous experimental reports of ferromagnetic order in Cr-doped GaN, e.g. [27-31] for $\mathrm{Cr}$ incorporated during growth and [32, 33] for implanted $\mathrm{Cr}$. This is, however, not unexpected. The literature of the last ten years on more extensively studied DMS materials, e.g. Mn-doped GaN, experienced a similar evolution. Despite the many theoretical predictions and experimental reports of high-temperature ferromagnetism, recent studies did not show any indication of high-temperature ferromagnetic order in single-phase Mn-doped GaN [11, 20, 22]. In fact, it is worthwhile discussing in more detail these two cases, $\mathrm{Cr}$ - and Mn-doped GaN, together. Two types of magnetic behavior have been comprehensively demonstrated in single-phase Mn-doped GaN: short-ranged AFM interactions between $\mathrm{Mn}^{2+}\left(3 d^{5}\right)$ moments [22] and ferromagnetic order of $\mathrm{Mn}^{3+}$ $\left(3 d^{4}\right)$ moments with a Curie temperature below $10 \mathrm{~K}$ [11]. The latter only occurs under stringent growth conditions to ensure epitaxial growth and, most importantly, to minimize the formation of compensating defects such as $\mathrm{N}$ vacancy

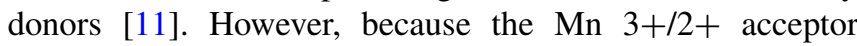
level is deep in the GaN bandgap, the associated holes are strongly localized. Since the coupling between distant moments via $p-d$ Zener exchange [57] requires itinerant holes, the observed Curie temperatures are very low $(<10 \mathrm{~K})$. More commonly, such stringent growth conditions are not satisfied and compensating donor defects are formed. The $\mathrm{Mn}$ impurities are thus incorporated as compensated $\mathrm{Mn}^{2+}$ and the $p-d$ Zener model does not apply. Consequently, only nearest cation neighbor AFM interactions are observed [22], which are likely to result from indirect superexchange between the Mn $3 d$ moments mediated by the $\mathrm{N} 2 p$ band. Indeed, significant hybridization of the Mn $3 d$ and $\mathrm{N} 2 p$ states has been observed [58]. This understanding of the magnetic interactions between $\mathrm{Mn}$ impurities in $\mathrm{GaN}$ in different charge states provides a good framework to interpret our results in the Cr-doping case. Chromium is incorporated in $\mathrm{GaN}$ as $\mathrm{Cr}^{3+}\left(3 d^{3}\right)[50,51]$, with only a donor level $(3+/ 4+)$ in the bandgap [59]. In the absence of $\mathrm{p}$-holes to mediate $p-d$ Zener exchange, our results show that the magnetic behavior of $\mathrm{Cr}^{3+}$ $\left(3 d^{3}\right)$ is very similar to that of $\mathrm{Mn}^{2+}\left(3 d^{5}\right)$ : short-ranged AFM interactions, which, as for $\mathrm{Mn}^{2+}$, are likely to result from indirect superexchange mediated by the $\mathrm{N} 2 p$ band. Indeed, the necessary hybridization of the $\mathrm{Cr} 3 d$ and $\mathrm{N} 2 p$ states has also been observed $[51,50]$.

As an alternative to the $p-d$ Zener models discussed in the previous paragraph, high-temperature ferromagnetism in wide-gap DMS materials has also been predicted on the basis of bound magnetic polaron (BMP) models [35]. Here, the magnetic moments interact via electrons associated with a defect impurity band. Since these defect electrons occupy large orbits, each defect can interact with several dopant moments and form a magnetic polaron. Ferromagnetism results from the percolation of such polarons. However, the exchange energy density necessary to produce hightemperature ferromagnetism at a few per cent doping corresponds to a magnetic exchange much stronger than that observed in the strongest of the known ferromagnetic materials [36]. One can thus expect that the critical temperatures associated with this type of magnetic order are well below room temperature.

Our results support the view that the source of hightemperature ferromagnetism often observed in transitionmetal-doped GaN may be non-intrinsic, i.e. associated with ferromagnetic precipitates or contaminations. This is in line with a very recent overview of the last ten years of DMS research, which concluded that little proof has been found of high-temperature ferromagnetism in wide-gap DMS materials [1].

\section{Conclusions}

In summary, we have experimentally demonstrated the absence of ferromagnetic order in Cr-implanted $\mathrm{GaN}$, from $300 \mathrm{~K}$ down to $2 \mathrm{~K}$, for $\mathrm{Cr}$ fractional concentrations between 0.005 and 0.35 . We have shown that the magnetic interactions between $\mathrm{Cr}$ moments in GaN are, in fact, AFM. Strong AFM interactions between the $\mathrm{Cr}$ atoms in nearest cation neighbor complexes result in $\mathrm{Cr}$ moment quenching (compensation), i.e. a decrease of the effective moment per $\mathrm{Cr}$ atom, with increasing concentration. Uncompensated $\mathrm{Cr}$ atoms behave as paramagnetic moments perturbed by AFM interactions in an anisotropic crystal field. Although minimally, thermal annealing affects the magnetic behavior, possibly due to an interplay between defect annealing and $\mathrm{Cr}$ aggregation. 
These results suggest that neither type of model of ferromagnetism proposed for dilute magnetic semiconductors, i.e. $p-d$ Zener exchange or BMPs, operates in $\mathrm{Cr}$-implanted $\mathrm{GaN}$. Together with recent reports on well-characterized Mn-doped GaN, our study supports the view that the high-temperature ferromagnetism observed in transitionmetal-doped GaN may be non-intrinsic.

\section{Acknowledgments}

This work was supported by the Research FoundationFlanders (FWO), the SPIRIT (Support of Public and Industrial Research using Ion Beam Technology) project (contract no. 227012), KULeuven projects GOA/2009/006 and INPAC EF/05/005 and the IUAP P6/42 program. L M C Pereira acknowledges the scholarship SFRH/BD/35761/2007 granted by the Portuguese Foundation for Science and Technology (FCT). T Som acknowledges the FWO for a fellowship.

\section{References}

[1] Dietl T 2010 Nature Mater. 9965

[2] Matsumoto Y, Murakami M, Shono T, Hasegawa T, Fukumura T, Kawasaki M, Ahmet P, Chikyow T, Koshihara S and Koinuma H 2001 Science 291854

[3] Pearton S J, Abernathy C R, Norton D P, Hebard A F, Park Y D, Boatner L A and Budai J D 2003 Mater. Sci. Eng. R 40137

[4] Prellier W, Fouchet A and Mercey B 2003 J. Phys.: Condens. Matter 15 R1583

[5] Pearton S J, Heo W H, Ivill M, Norton D P and Steiner T 2004 Semicond. Sci. Technol. 19 R59

[6] Liu C, Yun F and Morkoc H 2005 J. Mater. Sci., Mater. Electron. 16555

[7] Dhar S, Brandt O, Ramsteiner M, Sapega V F and Ploog K H 2005 Phys. Rev. Lett. 94037205

[8] Pan H, Yi J B, Shen L, Wu R Q, Yang J H, Lin J Y, Feng Y P, Ding J, Van L H and Yin J H 2007 Phys. Rev. Lett. 99127201

[9] Venkatesan M, Fitzgerald C B and Coey J M D 2004 Nature 430630

[10] Kittilstved K R, Liu W K and Gamelin D R 2006 Nature Mater. 5291

[11] Sarigiannidou E, Wilhelm F, Monroy E, Galera R M, Bellet-Amalric E, Rogalev A, Goulon J, Cibert J and Mariette H 2006 Phys. Rev. B 74041306

[12] Abraham D W, Frank M M and Guha S 2005 Appl. Phys. Lett. 87252502

[13] Ney A, Kammermeier T, Ney V, Ollefs K and Ye S 2008 J. Magn. Magn. Mater. 3203341

[14] Garcia M A, Fernandez Pinel E, de la Venta J, Quesada A, Bouzas V, Fernandez J F, Romero J J, Martin Gonzalez M S and Costa-Kramer J L 2009 J. Appl. Phys. 105013925

[15] Pereira L M C, Araujo J P, Van Bael M J, Temst K and Vantomme A 2011 J. Phys. D: Appl. Phys. 44215001

[16] Sun Z, Yan W, Zhang G, Oyanagi H, Wu Z, Liu Q, Wu W, Shi T, Pan Z, Xu P and Wei S 2008 Phys. Rev. B 77245208

[17] Zhou S et al 2008 J. Appl. Phys. 103023902

[18] Zhou S, Potzger K, von Borany J, Grotzschel R, Skorupa W, Helm M and Fassbender J 2008 Phys. Rev. B 77035209

[19] Zhou S, Potzger K, Kuepper K, Grenzer J, Helm M, Fassbender J, Arenholz E and Denlinger J D 2008 J. Appl. Phys. 103043901

[20] Stefanowicz W et al 2010 Phys. Rev. B 81235210
[21] Ney A, Kammermeier T, Ollefs K, Ye S, Ney V, Kaspar T C, Chambers S A, Wilhelm F and Rogalev A 2010 Phys. Rev. B 81054420

[22] Granville S, Ruck B J, Budde F, Trodahl H J and Williams G V M 2010 Phys. Rev. B 81184425

[23] Ney A, Ollefs K, Ye S, Kammermeier T, Ney V, Kaspar T C, Chambers S A, Wilhelm F and Rogalev A 2008 Phys. Rev. Lett. 100157201

[24] Sati P, Deparis C, Morhain C, Schafer S and Stepanov A 2007 Phys. Rev. Lett. 98137204

[25] Xu Q et al 2009 J. Phys. D: Appl. Phys. 42085001

[26] de Carvalho H B et al 2010 J. Appl. Phys. 108033914

[27] Hashimoto M, Zhou Y K, Kanamura M and Asahi H 2002 Solid State Commun. 12237

[28] Park S E, Lee H J, Cho Y C, Jeong S Y, Cho C R and Cho S 2002 Appl. Phys. Lett. 804187

[29] Zhou Y K, Hashimoto M, Kanamura M and Asahi H 2003 J. Supercond. 1637

[30] Kim J J, Makino H, Sakurai M, Oh D C, Hanada T, Cho M W, Yao T, Emura S and Kobayashi K 2005 J. Vac. Sci. Technol. B 231308

[31] Singh R K, Wu S Y, Liu H X, Gu L, Smith D J and Newman N 2005 Appl. Phys. Lett. 86012504

[32] Lee J S, Lim J D, Khim Z G, Park Y D, Pearton S J and Chu S N G 2003 J. Appl. Phys. 934512

[33] Wang J Q, Chen P P, Guo X G, Li Z F and Lu W 2005 J. Cryst. Growth 275393

[34] Pipeleers B, Hogg S M and Vantomme A 2005 J. Appl. Phys. 98123504

[35] Coey J M D, Venkatesan M and Fitzgerald C B 2005 Nature Mater. 4173

[36] Coey J M D, Stamenov P, Gunning R D, Venkatesan M and Paul K 2010 New J. Phys. 12053025

[37] Wang M J, Yuan L, Cheng C C, Beling C D and Chen K J 2009 Appl. Phys. Lett. 94061910

[38] Kucheyev S O, Williams J S, Jagadish C, Zou J and Li G 2000 Phys. Rev. B 627510

[39] Vantomme A et al 2001 Nucl. Instrum. Methods Phys. Res. B 175148

[40] Jiang W and Weber W J 2006 Nucl. Instrum. Methods Phys. Res. B 242431

[41] Kucheyev S O, Williams J S, Zou J, Jagadish C and Li G 2001 Appl. Phys. Lett. 781373

[42] Vanvecht J A 1973 Phys. Rev. B 71479

[43] Williams J S 1998 Mater. Sci. Eng. A 2538

[44] Gu L, Wu S Y, Liu H X, Singh R K, Newman N and Smith D J 2005 J. Magn. Magn. Mater. 2901395

[45] Nord J, Nordlund K, Pipeleers B and Vantomme A 2003 Mater. Sci. Eng. B 105111

[46] Biersack J P and Haggmark L 1980 Nucl. Instrum. Methods $\mathbf{1 7 4} 257$

[47] Kucheyev S O, Williams J S, Zou J, Jagadish C and Li G 2001 Nucl. Instrum. Methods Phys. Res. B 175214

[48] Bonanni A et al 2008 Phys. Rev. Lett. 101135502

[49] Ney A, Harris Jr J S and Parkin S S P 2006 J. Phys.: Condens. Matter 184397

[50] Takeuchi T et al 2004 Phys. Rev. B 70245323

[51] Hashimoto M, Emura S, Tanaka H, Honma T, Umesaki N, Hasegawa S and Asahi H 2006 J. Appl. Phys. 100103907

[52] Behringer R E 1958 J. Chem. Phys. 29537

[53] Cui X Y, Medvedeva J E, Delley B, Freeman A J and Stampfl C 2007 Phys. Rev. B 75155205

[54] Buschow K H J and De Boer F R 2004 Physics of Magnetism and Magnetic Materials (New York: Kluwer) p 24

[55] Hong J 2008 J. Appl. Phys. 103063907

[56] Xu B and Pan B C 2009 J. Appl. Phys. 105103710

[57] Dietl T, Ohno H, Matsukura F, Cibert J and Ferrand D 2000 Science 2871019

[58] Wang D, Zhang X, Wang J and Koide T 2009 Solid State Commun. 149192

[59] Gerstmann U, Blumenau A T and Overhof H 2001 Phys. Rev. B 63075204 